\title{
Context-aware Selection in Multicast Environments
}

\author{
Nuno Coutinho ${ }^{1}$, Tiago Condeixa ${ }^{1}$, Rui Valbom $^{1}$, Susana Sargento $^{1}$, Augusto Neto ${ }^{1} 2$ \\ ${ }^{1}$ Instituto de Telecomunicações, University of Aveiro, Portugal \\ \{nunocoutinho,tscondeixa,rui.valbom,susana\}@ua.pt, augusto@av.it.pt \\ ${ }^{2}$ Instituto de Informática, Universidade Federal de Goiás, Brazil \\ augusto@inf.ufg.br
}

\begin{abstract}
Personalization and group communications have always been conflicting approaches. From one side, the user wants to get the services with their specific characteristics. From the other side, group communications imply the support of the same service, with the same characteristics, by a group of users. To simultaneously enable personalization and profit from the resources improvements provided by group-based communications, these groups need to be defined based on the users requirements, services and network information, which we denote as 'context'. Based on this context for group definition, it is possible to adapt the content to the groups of users or even re-group them according to common features. This paper addresses the network part of the problem: based on the context information, we define an intelligent network selection, both for the access and core, respectively to efficiently choose the attachment points and the multicast trees for groups of users. This selection approach will improve the efficiency of the architecture in delivering the multiparty services. The simulation results of the proposed approach show that performing a wise network selection based on context increases overall network efficiency while still providing a personalized multiparty content delivery.
\end{abstract}

Index Terms-Context-awareness, Multicast, Mobility, Multiparty, Heterogeneity, Scalability.

\section{INTRODUCTION}

Increasing interest and popularity of group-based multimedia services propelled the research towards the development of novel solutions that make better use of operators resources while meeting the strict requirements of current and future applications. Service providers are also looking for new ways to differentiate their offer and so increase their revenues. Service personalization may be an added value for operators, although being a challenging goal considering group communication environments. Thus, future networks must be able to wisely use the available information and provide the services to users' needs, capabilities and preferences. Nevertheless, context-aware networks face several challenges: from one side, context (e.g. preferences, location, mobility, resources) may be used to select the best networks for the groups of services; from the other side, any context change may require a complete restructuring of the network and its sessions.

Group communication over heterogeneous environments increases the difficulty in deploying network selection. Considering different users interests, devices and networks characteristics and capabilities, it turns difficult to provide the same multicast session content to all interested users fulfilling all requirements (session, network and user group) at the same time. Moreover, the unpredictable occurrence of network events (link failures or user handover) may affect session quality. Thus, the session characteristics need to be adapted to user/network capabilities.

In order to overcome the requirements described hereinabove, we propose the efficient grouping of interested users according to their current context, such as terminal capabilities, network conditions, location, etc. For instance, the same session content can be sent simultaneously to sub-groups taking different codecs to improve network capabilities. Moreover, resilience can be improved through grouping approach, where only some sub-groups can be adapted so as to avoid affecting all interested users. Thus, we claim that efficient grouping mechanism is essential to improve network selection aiming at maintaining groups of mobile users always best connected while increasing network resources management efficiency.

The work addressed in this paper focuses on the access and core intelligent selection based on context information from the network- and user-side, as well as the specification of an efficient mechanism of grouping/sub-grouping of mobile users supporting network/user context. Thus, our goal is to increase the experience perceived by the user, offering contextaware multiparty services, while optimizing network revenues. The outcomes of the simulated approach show that contextawareness improves the overall network performance while providing a personalized multiparty content delivery.

This paper is organized as follows. The related work is presented in Section II. Section III describes the architecture and its functionalities, with more emphases on the network elements. Section IV introduces the core and access network selection schemes developed. Section V presents the evaluation of the architecture. Finally, Section VI concludes the paper and introduces the future work.

\section{RELATED WORK}

Context-awareness is crucial to endow NGNs with dynamic adaptability to user and environment characteristics. Service personalization may be influenced by a wide variety of context, which as defined by [1], is any information that can be used to characterize an entity. Making available more context information and understanding how it can be effectively used, it is possible to increase service flexibility and support intelligent personalization. Thus, it is essential to develop frameworks to gather, manage and disseminate contextual information, as well as to react promptly to context changes. Context-aware environments were addressed in several research projects, such 
as the NEON project from Nokia Siemens [2], the contextaware personalization aspect [3] of IST-Daidalos architecture, and the approach of IST-Ambient Networks [4].

Mentioned architectures (excepting [4]) only use context information to favor service personalization, rather than a more global employment of the knowledge acquired in a better network resource management while maintaining user Quality of Experience (QoE). This is even harder to accomplish in groupbased communications, where IP Multicast appears as the most suitable technology to save resources. Its design issues [5], architecture complexity and open service model, raised some concerns with the operators regarding its commercial viability. However, proposals as the Hop by Hop Multicast Routing protocol $(\mathrm{HBH})$ [6], are focused on the progressive deployment of multicast allowing the interoperability of unicast and multicast enabled networks. Moreover, scalability concerns were addressed in [7], improving the work of [6] by reducing forwarding states.

Despite these efforts, in order to make multicast suitable for next generation applications, it is mandatory the integration with QoS mechanisms, as the class-based QoS and IP multicast integration proposed by [8] and the resource reservation protocol developed by [9]. An integration of multicast and Multi-protocol Label Switching (MPLS) technologies is also introduced addressed by [10], building multicast trees over MPLS networks, which increases multicast scalability and bandwidth management efficiency. However, these works attempt to select and reserve the overall path, which stills not very scalable regarding the constraints involved in the construction of multicast trees.

Finally, since the main goal of our framework is to provide personalized content delivery to multiple mobile users, a key feature in the architecture is an intelligent access network selection. Given the heterogeneity of wireless technologies and mobile devices, the service provided is most of all limited by the capabilities of access networks, users and mobile devices. Although there are several approaches in the literature, [11][12][13][14], they mostly deal with unicast traffic and do not consider grouping and sub-grouping of users to provide group-based selection schemes.

\section{Context-Aware Multiparty Transport}

C-Cast project provides the basic architecture for our work. Its architecture relies on several functionalities that combine context-awareness, session and network multiparty technologies. In the envisioned architecture, any change of context may influence sessions established, requiring their renegotiation and consequently the reconfiguration of the network. Moreover, mobility will add a new dynamic to future networks, rising the necessity to efficiently deal with constant changing environments. Fig. 1 presents the general architecture, briefly explaining in the following the function of each component, giving more emphasis to the networking elements.

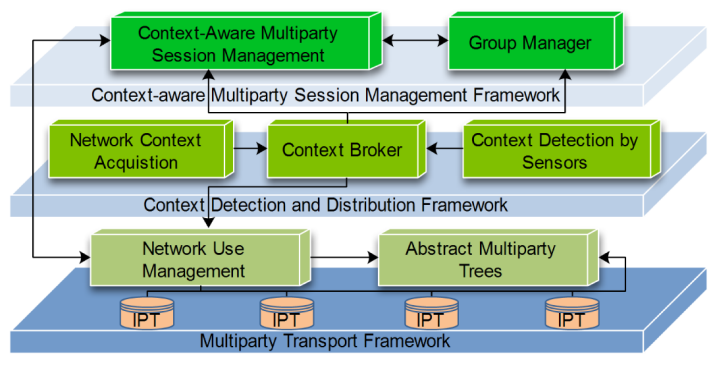

Figure 1. C-CAST functional architecture

\section{A. Context Detection and Distribution Framework}

This set of modules supports the context detection part, from the network, terminals and applications, using sensors throughout the network, the Context Providers. The context information is then periodically gathered in a repository, called Context Broker (CB), which can be described as the context database of the network. CB also delivers information to the network decision entity, denoted as the Network Use Management (NUM) to perform network selection (including grouping and sub-grouping decisions), and multicast resource allocation for the group communications.

\section{B. Context-Aware Multiparty Session Management}

The session management works as an overlay between applications and networks, being responsible for session control: session establishment, re-negotiation and termination. Additionally, it creates the session context, which describes the characteristics of the sessions, such as codec, delay, loss rate, jitter and identification of the members of the session's group. The Group Manager (GM) entity provides the first grouping of users, taking into account capabilities and environment context. Members may be then split into sub-groups, aiming the best fit between the different media encodings available of the content, user's device capabilities and network resources available. In this work, we will focus in the network level sub-grouping, providing resources aggregation and QoS in personalized and multicast context-aware communications.

\section{Context-Aware Multiparty Transport Framework}

This framework provides the context-aware multiparty transport, performing network selection to the groups of users, both for the access and core part, sub-grouping of users based on network context, and an efficient and scalable network adaptation to context changes. It also supports the allocation of resources to provide multicast services with delivery guarantees. This framework comprises the following elements.

1) Network Use Management - NUM: NUM can be described as the network brain of the architecture, since it provides intelligent context-aware network selection to select subpaths for the multicast trees, in the core and access networks, and provides resource allocation for these sub-paths. The main purpose of NUM is to maintain all multihomed terminals always best connected, offering the best combination of users and services through a heterogeneous system while it aims 
at achieving enhanced network capacity and performance. In the access network, NUM provides network selection based on context information, combining user, environment, session and network contexts to determine the best decisions in terms of access to the users (this decision will also build the subgroups of users). In the core network, NUM chooses the best sub-paths that will build the multicast trees. Consequently, it is expected to achieve a more uniform distribution of the load between the different radio access technologies and core nodes, while satisfying users' requirements and preferences. Although NUM is a central element, scalability is achieved through the adoption of a hierarchical network multiparty control framework that distributes local functionalities throughout network elements, such as the Overlay Nodes (ONs) and IP Transport (IPT), as will be described in the next sub-sections.

2) Abstract Multiparty Trees - AMT: The concept of abstract transport multiparty trees operates on top of the IP network layer, aiming at allowing general transport control in multicast trees hiding the network dynamics, consisting in defining overlay transport structures called Abstracted Multicast Tree (AMT). Considering the slowly employment of multicast by operators due to many reasons [5], unicast and multicast enabled networks will coexist in future networks. Thus, AMTs allow end-to-end multicast content transport over network segments with different transport technologies, also providing independence between source and listener trees. This independence will enable the easy support of local reconfigurations and its integration with $\mathrm{QoS}$ for the multicast trees.

Local network segments are called sub-AMTs, where all nodes (edge and core) composing a sub-AMT implement the same transport technology. NUM coordinates the edges of each sub-AMT, the ONs introduced in the previous section, which may be any core node endowed with more resources and functionalities. This level of freedom is justified by the fact that the definition and selection of these nodes have a potential impact in the network performance, as we will assess in Section V. In the scope of a sub-AMT: (i) ingress ON is viewed as a session source; (ii) egress ONs as leaf nodes (or listeners); (iii) and core nodes remain simple by mainly deploying IP forwarding operations.

Following this strategy, the processes of resource reservation and network reconfigurations are of decreased complexity in the overall network. With sub-AMTs, resources and QoS are assigned per sub-AMT. Moreover, since the path will be broken into several sub-AMTs, local reconfigurations can be done inside each sub-AMT, coordinated by the ONs, without involving the NUM and the other sub-AMTs; this will increase scalability and efficiency of the reconfiguration process. NUM only needs to work on the network reconfiguration if changes are required to the ONs of the sub-AMT. As ONs limit the scope of the sub-AMTs, the level of flexibility and reconfiguration capability increases with the use of more ONs, specially in nodes closer to edges, since it is the area most susceptible to events and changes (new terminal arrives, moves or leaves).

Fig. 2 shows an example of a network scenario with

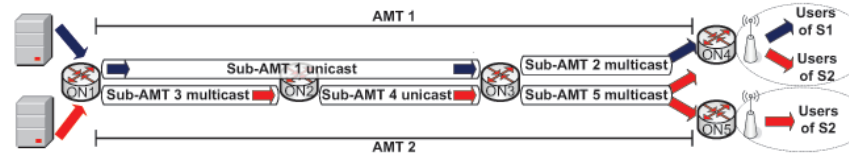

Figure 2. End-to-end AMTs and interiors Sub-AMTs.

different AMTs to supply multicast transport for sessions S1 and S2. The support of different transport technologies (subAMTs 1 and 4) is assured by ONs 1 and 2, performing proxy functions to mediate overlay connections and to control the QoS of the sub-AMT independently from the others. In the $\mathrm{ON} 3$, multicast addressing is again employed. In this way, the changes that occur in the transport are transparent for the users. In case there are no core routers with the ability to perform ON functions, all nodes would have the same functionalities and the network reconfigurations would span through all nodes in the multicast paths. This would obviously decrease the level of flexibility and reconfiguration of the transport.

3) Internet Protocol Transport - IPT: The IPT component aims to allocate network resources, building sub-AMTs in the paths between ONs, to support multiparty content delivery with QoS guaranteed over the group session lifetime. IPT is based on the existing Multi-Service Resource Allocation (MIRA) [9] proposal to support scalable QoS allocation and mapping, efficient IP multicast control, and fast resilience operations. MIRA assures the quality level requested for each flow of a multi-user session, by adjusting the resources of the selected Class of Service (CoS). We provide the construction of QoS-aware multicast trees for each session-flow by manipulating the Multicast Routing Information Base (MRIB). The $\mathrm{CoS}$ bandwidth and the MRIB are updated in a single-pass operation from the ingress to the egress located in the direction of the access-router of the receiver. In the ingress-router and in each interior router, the configuration of the selected $\mathrm{CoS}$ is done in the outgoing interface indicated by the unicast RIB. After configuring the CoS, the MRIB is updated with the IP address of the previous visited router. In an egress-router, the selected $\mathrm{CoS}$ are configured taking into account the service level agreements (SLS) established with the neighbor network, and PIM-SSM is triggered after configuring the MRIB. IPT supports a distributed per-class resource control, thus whereas session establishment can be requested in a per-flow basis, resources are configured per-aggregate. IPT provides seamless resilience operations, since it attempts to reconfigure multicast trees between ONs inside the sub-AMTs, without changing multicast groups address and ONs.

\section{SELECTION}

The selection scheme proposed in this paper aims to improve the efficiency of the multiparty content distribution based on the context information available. The adopted strategy considers different schemes for Access and Core network selection, Fig. 3, since the involved constraints for both parts are different, and a unique algorithm involving all would be infeasible and most of all not scalable. Thus, two schemes 


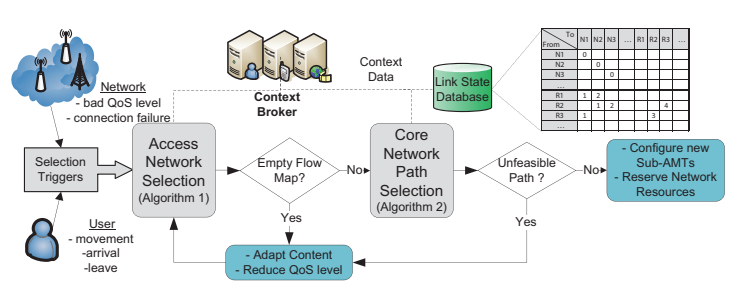

Figure 3. Overall Context-aware Selection Scheme

with different inputs and outputs were developed, starting with the access network selection to obtain the most suitable Access Point (AP) for the user. This AP will work as a ON in the access network. This procedure attempts to achieve the best compromise between user preferences/requirements and network resources, giving a special attention to the group/subgrouping concept. Based on the AP chosen, the core network path selection is performed to determine the best feasible path between session content source and the selected AP, through multiple sub-AMTs between ONs, considering network QoS metrics and available bandwidth. Both schemes will be detailed in the following subsections.

\section{A. Access Selection}

The access network selection scheme adopted is described by its pseudo-code in Algorithm 1 (the correspondent unicast base algorithm was proposed in [12]). It was designed to determine a ranked list of candidate APs, with sufficient resources and to which the terminal is able to connect. Since the architecture proposed in this work aims a context-aware multiparty content delivery, an integration with QoS mechanisms and multicast constraints is required. Thus, it would be on the best interest of operators to aggregate in the same AP users that share the same content source, $\mathrm{CoS}$ or other features (e.g. device capabilities, location, codecs).

In this work, we propose to include in the access selection process the constraints depicted in Table I: User Preference, Cost, Sub-grouping, CoS and Bandwidth Allocation. Nevertheless, a wide variety of parameters could also be used, such as signal strength, Bit Error Rate or location. Table I values span between 0 and 100 , the higher representing the better value. The User Preference value of each AP is empirically assigned by the user, possibly taking into account its background experience. The Cost parameter is directly associated with the access price. Bandwidth Allocation is the result of a simple function that returns the value 100 when any traffic flows through the AP and 0 if there are no resources available. Considering the Sub-grouping constraint, a higher value means that the AP is serving a low number of different groups and it may support a session of a different group. The $\mathrm{CoS}$ parameter has a similar function, it intends to aggregate traffic with the same $\mathrm{CoS}$, being assigned higher values for APs that already have flows with the same CoS. Table II holds, for each user profile, the weights that each one assign to the constraint involved in the access selection. These values

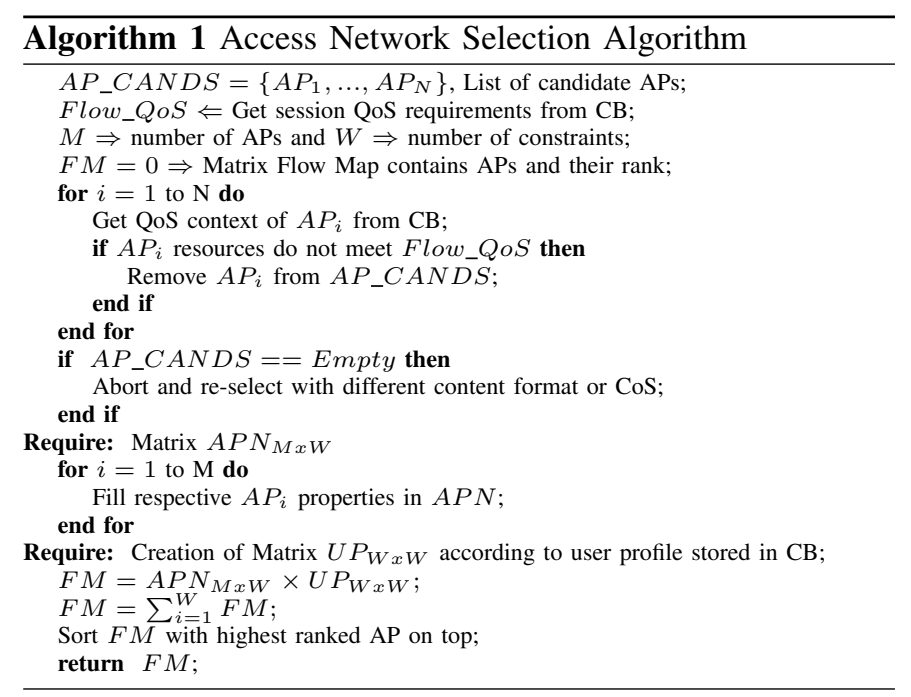

span between 0 and 1.5, allowing constraint differentiation according to each user.

The access selection algorithm, Algorithm 1, starts by the obtaining from the $\mathrm{CB}$ a list of APs $\left(A P \_C A N D S\right)$ in the range of the user. From the same repository, it also gets the session QoS demands (Flow_QoS) mapped in bandwidth, delay and loss rate requirements. This information is then crossed with the QoS context of each AP candidate, executing a simple admission control mechanism. Then, the matrix $A P N$ is built based on the context of each AP that passes the admission mechanism, which final form will be similar to Table I. After that, it is also created the matrix $U P$ with the user context relative to each of the constraints involved (based on Table II). Combining both matrices, and after some simple matrix operations, it is obtained the Flow Map $(F M)$ with the information about the most suitable APs regarding user and communication context.

Fig. 4 depicts a case scenario for a better understanding of sub-grouping and $\mathrm{CoS}$ parameters. Consider that Mobile Terminal 2 (MT2) wants to join Session 1, already subscribed by MT1, being within the range of both APs. In this case, the sub-grouping value assigned to AP1 is greater than for

Table I

AP PROPERTIES AND RESPECTIVE VALUES.

\begin{tabular}{c|c|c|c|c|c}
$\begin{array}{c}\text { Access } \\
\text { Technology }\end{array}$ & $\begin{array}{c}\text { User } \\
\text { Preferences } \\
\text { (static) }\end{array}$ & $\begin{array}{c}\text { Cost } \\
\text { (static) }\end{array}$ & $\begin{array}{c}\text { Sub-grouping } \\
\text { (dynamic) }\end{array}$ & $\begin{array}{c}\text { CoS } \\
\text { (dynamic) }\end{array}$ & $\begin{array}{c}\text { Bandwidth } \\
\text { Allocation } \\
\text { (dynamic) }\end{array}$ \\
\hline \hline WiMAX 1 & 100 & 30 & 90 & 90 & 50 \\
\hline UMTS 1 & 70 & 50 & 60 & 50 & 100 \\
\hline Wi-Fi & 80 & 80 & 0 & 0 & 90 \\
\hline WiMAX 2 & 100 & 30 & 30 & 70 & 10
\end{tabular}

Table II

WEIGHT DISTRIBUTION ACCORDING TO USER PROFILES.

\begin{tabular}{c|c|c|c|c|c}
$\begin{array}{c}\text { User } \\
\text { Profile }\end{array}$ & $\begin{array}{c}\text { User } \\
\text { Preferences }\end{array}$ & $\begin{array}{c}\text { Monetary } \\
\text { Cost }\end{array}$ & Sub-grouping & CoS & $\begin{array}{c}\text { Bandwidth } \\
\text { Allocation }\end{array}$ \\
\hline \hline Business Man & 0.5 & 1.0 & 1.5 & 1.0 & 1.0 \\
\hline Gamer & 1.5 & 0.5 & 1.0 & 1.5 & 1.0 \\
\hline Groupie & 0.5 & 1.5 & 0.5 & 0.5 & 0.5
\end{tabular}




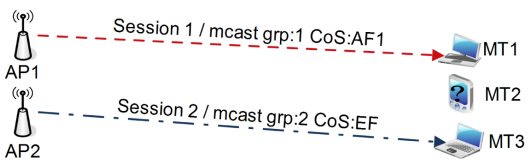

Figure 4. Example of a context-aware AP selection.

AP2, since is already flowing through AP1. If MT2 would like to receive a Session3 (mcast grp:3 CoS:EF), the CoS value for AP2 would be higher than for AP1, since AP2 already transmits a flow of the EF class. In case of MT2 desires to receive a Session 4 (mcast grp:4 CoS:AF2), although any AP have an AF2 class flow, the CoS value of AP1 will be higher than for AP2, since AP1 already transmits a similar class (AF1). However this value is smaller than in case of existing an AP transmitting a AF2 class flow.

\section{B. Core Selection}

Once selected the most suitable AP regarding communication needs and user context, the best path between content source and the chosen AP is selected. In our architecture this path is mapped in an AMT, as explained in Section III-C2, formed by a set of sub-AMTs bounded by ONs, where the AP is also considered as a $\mathrm{ON}$. We assume that the ONs are already defined in the network, being the subAMTs defined by the ONs that belong to the path selected. If we consider a network where all core nodes are capable of performing ON functionalities, we may then select them and construct sub-AMTs based on that, which would require a new intelligent selection scheme that is out of the scope of this work. However, we assess the impact of ONs' number and positioning in the network performance.

The core path selection scheme is presented in Algorithm 2. Available links in the network $(L)$ and their characteristics in terms of bandwidth available $(B W)$, routing cost $(C o s t)$ and mean delay (Delay) observed are retrieved from NUM's database, being essential that this information be updated before performing a selection. Another crucial input is a list (dst) of Egress ONs to which the content source will send traffic, including not only the AP previously selected but also

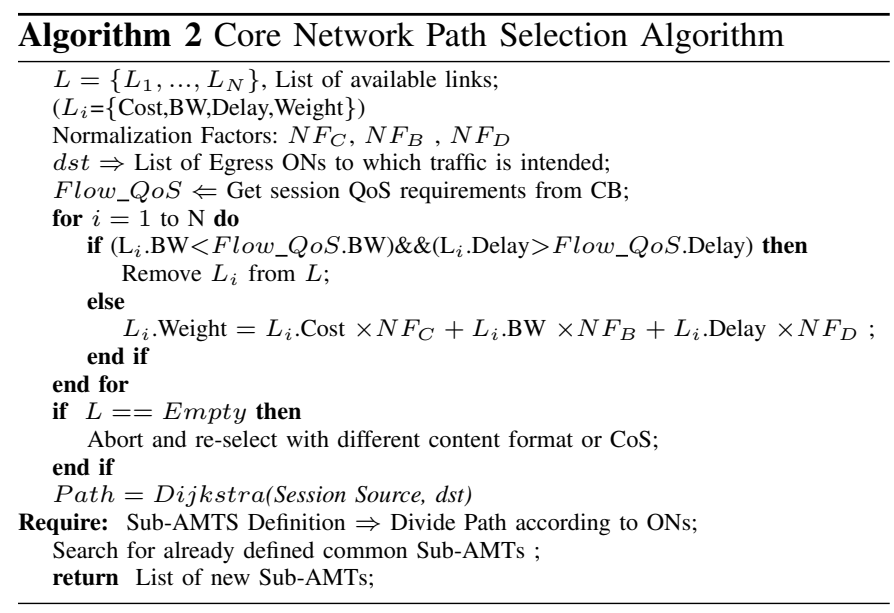

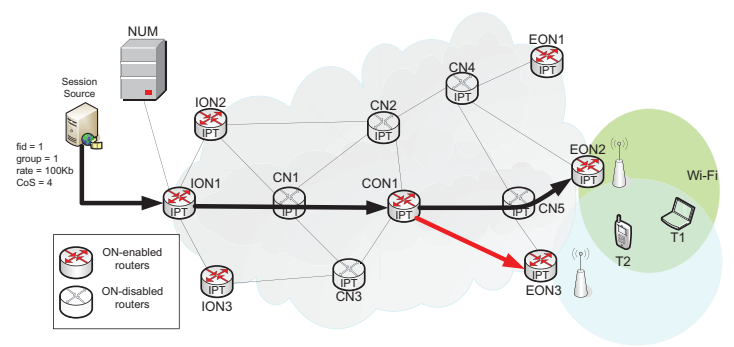

Figure 5. Example of one of the scenarios evaluated.

all the other APs that share the content source. Once again, considering session QoS demands (Flow_QoS) retrieved from $\mathrm{CB}$, an admission control is executed based on links' QoS context. To each admitted link, it is computed a cost (Weight) combining link's bandwidth available, delay and routing cost. Normalization factors $\left(N F_{C}, N F_{B}, N F_{D}\right)$ are associated to each of these parameters so that links' weights may be related with each other. Dijkstra's algorithm is then employed to compute the constrained shortest path to the respective APs. Based on the resultant path, and considering ONs within it, sub-AMTS are defined between two ONs. However, if there is a path previously selected for the same content source, some sub-AMTs already defined are very likely to be re-used, which saves network and resource configurations.

In both selection schemes, a situation can occur in which the network resources available are not sufficient to accommodate a new flow with its QoS required. In order to solve this issue, a possible solution could be the re-establishment of a new session with a different content format (codec) requiring less resources, as is presented in Fig. 3. At the network level, as long as the user accepts higher delays and losses, the session content could be distributed with a lower level of QoS supported by network resources.

\section{EVALUATION}

The architecture previously described was implemented in the Network Simulator 2 [15]. In order to assess its performance and robustness, it was created a flexible network topology regarding the number of ingress, egress and core nodes, core ON, APs, MTs, data sources and sessions. This way, we easily evaluate the response of the scheme implemented by varying the input parameters and generating different scenarios, as the one presented in Fig. 5. A different seed in each simulation changes the origin and destination nodes of the links in the scenario.

The fixed links in the core network were configured with a random delay between 1 and $2 \mathrm{~ms}$ and a bandwidth (BW) ranging between 5 and $7 \mathrm{Mbps}$. Two different traffic generators were randomly used: exponential traffic (EXP) and constant bit rate (CBR). All flows have a packet size of 1000 bytes and an average rate of $100 \mathrm{Kbps}$. The Class of Service (CoS) of each flow is randomly chosen, but it is guaranteed that more than a half of the traffic is Best Effort (BE) to simulate a real network. Moreover, six CoS were considered: signalling, 
Table III

INPUT PARAMETERS CONFIGURED IN SIMULATIONS

\begin{tabular}{|c|c|c|c|c|c|c|c|}
\hline & Ingress & Egress & Core & Source & MTs & Core ONs & APs \\
\cline { 1 - 5 } i) & 2 & 2 & 5 & 4 & 10 & 2 & $1-10$ \\
\cline { 1 - 5 } ii) & 2 & 2 & 6 & 2 & 15 & 3 & 7 \\
\hline iii) & 2 & 3 & 6 & 4 & 10 & $0-4$ & 7 \\
\hline iv) & 3 & &
\end{tabular}

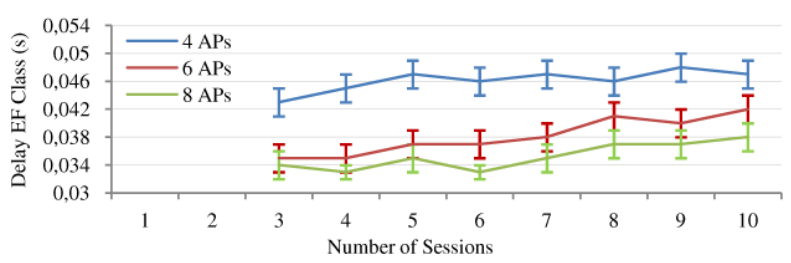

Figure 6. EF Class Delay per number of sessions.

routing, Expedited Forwarding (EF), BE, Assured Forwarding (AF) 1 and AF2.

\section{A. Influence of the APs' number}

In this section we assess the impact in the architecture response when varying the number of APs, considering resource saturation situations. We also show the admission control mechanism behaves under these situations. The network topology input parameters used are described in configuration i) of Table III. The number of users per session is $20 \%$ of the total users' number, where each session is composed by two flows. It is a major concern that each user receives its content according to the QoS requirements of the application. Hence, an efficient admission control must be performed to guarantee that each $\operatorname{CoS}$ has its own requirements fulfilled.

The results described on Fig. 6 show, as expected, that for a higher number of APs the delay is lower, due to the implicit load balance mechanism of the selection algorithm. Still, it is possible to observe the effects of the QoS provisioning mechanisms since the delay values do not increase much with the number of sessions, do to the admission control. Despite not presented, the overhead introduced is not significant (around $0.4 \%$ ) yet dependent of the number of network elements, in this case the number of APs.

\section{B. Access Network Selection Methods}

This evaluation shows the performance response of different access network selection methods. The "Basic" method represents the traditional AP selection based on the signal strength. The second one, "Sub-grouping Without CoS", treats all traffic as equal using a load balancing mechanism, user preferences and cost. The last method, "Sub-grouping With CoS", introduces the Sub-grouping and CoS in order to better manage traffic with different requirements. It also provides an admission control mechanism to prevent high priority traffic to be impaired.

The input parameters are the ones presented in the configuration ii) of Table III. Considering the results obtained for the different access strategies, Fig. 7 shows that the method "Sub-grouping With CoS" is obviously the better choice

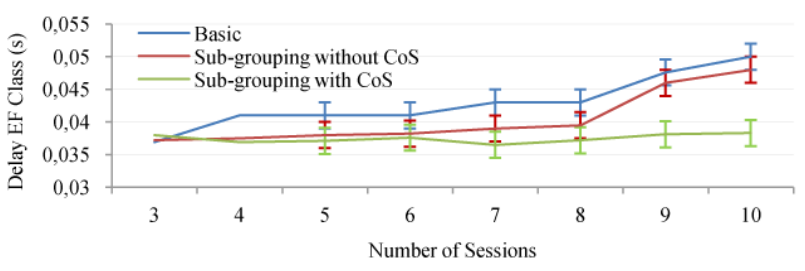

Figure 7. EF Class Delay for different access selection methods.

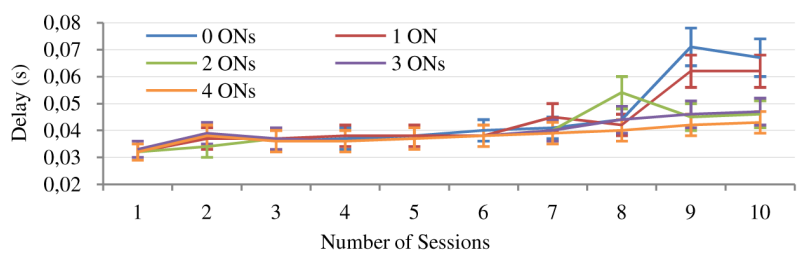

Figure 8. Delay dependent of ONs' number and number of sessions.

when dealing with EF class traffic. Moreover, the delay value maintains nearly constant due to the above mentioned admission mechanism. Comparing the "Basic" approach with the "Sub-grouping Without Cos" method, we may observe better delays for each one of the sessions' number simulated. These results show that, intelligently involving more information in the access selection process, it is possible to achieve better performances while maintaining user satisfaction.

\section{Influence of ONs}

The ONs are crucial components of the architecture proposed and, since they are directly connected to the creation of sub-AMTS, it is important to understand the impact of ONs' number in the core network performance. We implement the core selection algorithm in all these experiments. The network scenario considered contains, as inputs parameters, the values of the configuration iv) of Table III.

From the results obtained in Fig. 8, one may observe that packet delay decreases with the number of ONs introduced in the core network. Since the number of ONs is proportional to the number of sub-AMTs, as more sub-AMTs exist in the core, less reservations and/or releases are needed to deliver content to the mobile users. These results are more notorious in higher number of sessions, because it is likely that some sessions would use flows that already have paths reserved in the core network, only requiring an extension of the multicast tree. This way, the network reacts quickly to changes and in a scalable way, leading to lower delays. It should be stressed that after a certain point, increasing the number of ONs does not correspond to substantial improvements and may not justify collateral costs such as overhead.

As scalability and resilience are main concerns of the architecture proposed, it is important to assess the behavior of the components implemented regarding these issues. To perform this evaluation, we considered several scenarios with different number of ONs where a mobile terminal performs an handover. Thus, the network must be reconfigured to deliver content through the new AP. The number of reconfiguration 


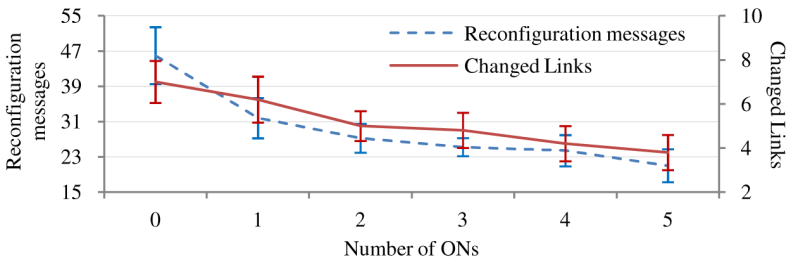

Figure 9. Impact of ONs in reconfiguration messages and changed links.

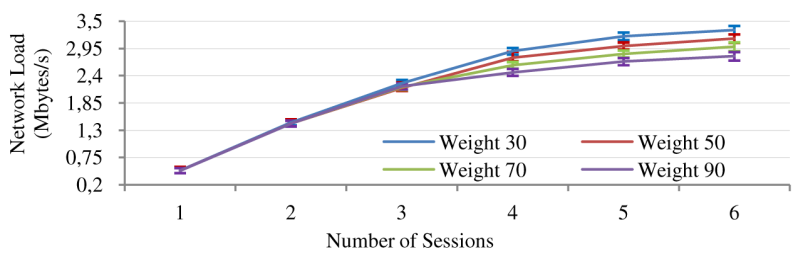

Figure 10. Network Load varying sub-grouping weight.

messages exchanged in this process and the links changed are presented in Fig. 9.

Observing the results obtained, one may conclude that the introduction of ONs in the core network increases architecture scalability, since both metrics decrease as the number of ONs increase. We may say that core ONs ease the reconfiguration process of the QoS-aware multicast tree, increasing the scalability of the architecture proposed and the AMTs concept. Since 5 ONs represents a core full of ONs, the results show that there are no benefits in using such a configuration of a core full of ONs, since the reconfiguration messages and changed links will tend to be the same and the overhead continues to increase. A balanced number of ONs and simple nodes in the core network is the most scalable solution.

\section{Sub-grouping}

The sub-grouping concept already described in section III.A allows two or more users to receive the same content but with different formats in order to a better adaptation to user capabilities and network conditions. From a multicast perspective, users are in the same group but with different subgroups. In our implementation, sub-grouping is also applied when a multicast group is divided between different APs in order to maintain the QoS correspondent to each CoS.

In this evaluation, users have different preferences for the sub-grouping parameter, being the network performance evaluated for different values of the sub-grouping weight. A high sub-grouping value means more users receiving content in the same AP within the same group. The network topology parameters considered for these simulations are described in the configuration iii) of Table III.

According to Fig. 10, as the sub-grouping weight increases, the network load decreases, which is more evident for a number of sessions higher than 3. Despite not presented, the overhead has a similar behavior. This is explained by the fact that with a high value assigned to the sub-grouping property, the same AP has more chances to be chosen and consequently the same core path. Thus, it is avoided unnecessary content replication, as long as the QoS requirements are satisfied. In fact, the algorithm should be designed to achieve a good compromise between acceptable delays and network load.

\section{COnclusions And Future Work}

In this paper we presented and evaluated a novel architecture able to provide personalized multiparty session content delivery to multiple mobile users. A special focus was given to the context-aware selection mechanisms, access and core networks, to provide an end-to-end context-aware service support. The evaluation of the implemented architecture shows the efficiency of the context-aware selection schemes, even for $\mathrm{QoS}$ purposes, as it is able to provided different QoS guarantees to several $\mathrm{CoS}$ in this multicast environment. The importance of introducing ONs in the core network was also evident once the scalability improvements that they may offer have direct impact in network performance when dealing with a high number of sessions. Considering the access network selection methods, we may conclude that using, in an intelligent way, more information in the decision process, better network performance may be achieved while satisfying user preferences. The sub-grouping functionality implemented was very simple, though it provides good results when avoiding data redundancy while ensuring a certain level of personalization and QoS.

We believe that this work is a first step towards overcoming demands for future internet services and technologies. Still, as future work we plan to develop both selection algorithms, by introducing self-management and autonomic concepts to achieve self-organized architectures in order to deal in a scalable way with the complexity and constant changes of the future mobile environments.

\section{REFERENCES}

[1] A. K. Dey, "Understanding and using context," Personal and Ubiquitous Computing, vol. 5, pp. 4-7, 2001.

[2] O. Riva et al., "A next generation operator environment to turn contextaware services into a commercial reality," in MDM, 2008.

[3] M. Williams et al., "Context-awareness and personalisation in the daidalos pervasive environment," in ICPS, July 2005, pp. 98-107.

[4] B. Mathieu et al, "Self-management of context-aware overlay ambient networks," in IFIP/IEEE IM, 21 2007-Yearly 25 2007, pp. 749-752.

[5] C. Diot et al., "Deployment issues for the ip multicast service and architecture," IEEE Network, vol. 14, no. 1, pp. 78-88, Jan/Feb 2000.

[6] L. H. M. K. Costa, S. Fdida, and O. Duarte, "Hop by hop multicast routing protocol," in ACM SIGCOMM, 2001, pp. 249-259.

[7] A. Boudani and B. Cousin, "Sem: a new small group multicast routing protocol," in ICT, Feb.-1 March 2003.

[8] R. Bless, K. Nichols, and K. Wehrle, "A lower effort per-domain behavior (pdb) for differentiated services," RFC Editor, 2003.

[9] A. Neto et al., "A resource reservation protocol supporting qos-aware multicast trees for next generation networks," in IEEE ISCC, July 2007.

[10] A. Boudani and B. Cousin, "Multicast tree in mpls network," in IEEE GLOBECOM, vol. 2, Nov.-2 Dec. 2005, pp. 6 pp.-.

[11] K. Pahlavan et al., "Handoff in hybrid mobile data networks," IEEE Personal Communications, vol. 7, no. 2, pp. 34-47, Apr 2000.

[12] V. Jesus, S. Sargento, and R. Aguiar, "Any-constraint personalized network selection," in IEEE PIMRC, Sept. 2008.

[13] A. Iera et al., "An access network selection algorithm dynamically adapted to user needs and preferences," in IEEE PIMRC, Sept. 2006.

[14] C. Prehofer, N. Nafisi, and Q. Wei, "A framework for context-aware handover decisions," in IEEE PIMRC, vol. 3, Sept. 2003.

[15] "The Network Simulator NS-2," http://www.isi.edu/nsnam/ns/. 